\title{
The value of double inversion recovery MRI sequence in assessment of epilepsy patients
}

\author{
Mohamed Abdelbar Abdelmaksoud Aly ${ }^{1 *} \mathbb{D}$, Tarek Mohamed Saleh ${ }^{1}$, Amr Mohamed Ahmed Elfatatry ${ }^{2}$ and \\ Moataz Mohamed Montasser ${ }^{1}$
}

\begin{abstract}
Background: The double inversion recovery (DIR) pulse sequence was introduced several years ago and since that it grew important value in clinical neuroimaging. We aimed to assess the added value of double inversion recovery in evaluation of epileptic patients.

Results: In mesial temporal sclerosis, the measured contrast parameters (SNR, CR, CNR and Al) were found to be significantly higher in DIR than in FLAIR and T2 sequences. In cases of focal cortical dysplasia, significantly higher CNR and Al in DIR than in T2 and FLAIR. Also DIR showed higher detection of the increased cortical thickness and cortical signal intensity than the T2 and FLAIR sequences. In tuberous sclerosis cases, the DIR showed higher visibility of the lesions than the T2 and FLAIR. Also DIR showed higher ability to detected grey-white matters junction blurring.

Conclusions: Our study concluded that the greatest value of the double inversion recovery sequence is its higher ability in detecting multiple characteristics of the lesions in a one sequence.

Keywords: Double inversion recovery, Epilepsy, Mesial temporal sclerosis, Focal cortical dysplasia, Malformations of cortical development
\end{abstract}

\section{Background}

Epilepsy is a common chronic neurological brain condition that has wide range of causes with multiple influencing factors in most cases [1]. The MRI appears as a major player in its work up, however, a specifically tailored MRI protocol [2]. The Double inversion recovery (DIR) is appearing in the last few years to have a very important role in the neuroimaging as an excellent grey matter imaging modality [3].

Of the major causes of epilepsy appear the mesial temporal sclerosis and malformations of cortical development, as two of the most common causes of epilepsy among adult and pediatric age groups respectively [4, 5]. Mesial temporal sclerosis (MTS) is characterized by neuronal loss and gliosis affecting selective parts of the

\footnotetext{
${ }^{*}$ Correspondence: mohd.abdelbr@gmail.com

1 Department of Radiodiagnosis, Faculty of Medicine, University

of Alexandria, Alexandria, Egypt

Full list of author information is available at the end of the article
}

hippocampal formation [6]. On the other hand, malformations of cortical development (MCDs) are a very wide and diverse range of disorders presented by multiple MR imaging features [7]. They include focal cortical dysplasias (FCDs), tuberous sclerosis (TSc), heterotopia, and polymicrogyria among other disorders $[5,7,8]$.

MTS may represent a diagnostic challenge on MRI, as in the early stage, where the main findings of hippocampal sclerosis (the atrophy and T2 hyperintensity) are subtle $[6,9]$.

Also, some of the malformations of the cortical development (MCDs) are difficult to characterize based on the current MRI modalities and they can mimic each other. So we conducted this study hoping that it can increase the efficacy of MRI in detecting the epileptogenic lesions so helping patients getting better management.

\section{Methods}

The study included 25 patients (18 females and 7 males). Their ages ranged between 1 and 57 years. 


\section{Inclusion criteria}

25 consecutive patients with history of primary idiopathic epilepsy and their MRI revealed epileptogenic lesions were prospectively included in the study. All age groups were included.

\section{Exclusion criteria}

Patients with space occupying lesions, inflammatory pathology and vascular malformations.

\section{All patients were subjected to}

1. Thorough history taking.

2. Conventional MRI epilepsy protocol, including: All patients in this study were imaged using 3T magnet MRI machines; GE Discovery closed configuration whole body scanner using a standard head coil.

A. Three dimensional (3D) T1 weighted image utilizing a repetition time (TR) of $8 \mathrm{~ms}$, an echo time (TE) of $3 \mathrm{~ms}$, an isometric slice thickness of $1 \mathrm{~mm}$, $\mathrm{FOV}=230 \times 180 \mathrm{~mm}$.

B. Axial and coronal T2 weighted image utilizing a repetition time (TR) of $4000 \mathrm{~ms}$, an echo time (TE) of $100 \mathrm{~ms}$, a slice thickness of $5 \mathrm{~mm}$, $\mathrm{FOV}=230 \times 180 \mathrm{~mm}$.

C. Three dimensional (3D) FLAIR utilizing a repetition time (TR) of $7500 \mathrm{~ms}$, an echo time (TE) of $110 \mathrm{ms,}$ TI of $2000 \mathrm{~ms}$, an isometric slice thickness of $1 \mathrm{~mm}$ and $\mathrm{FOV}=230 \times 180 \mathrm{~mm}$.

3. Double inversion recovery (DIR) sequence:

We used a three dimensional double inversion recovery (DIR) sequence utilizing a repetition time (TR) of $7000 \mathrm{~ms}$, an echo time (TE) of $80 \mathrm{~ms}$, inversion times (TI) of $3400 / 325 \mathrm{~ms}$, a slice thickness of $1 \times 1 \times 2 \mathrm{~mm}$ and $\mathrm{FOV}=230 \times 180 \mathrm{~mm}$.

\section{Image analysis}

All images were analyzed by two experienced neuroradiologists. To test the impact of DIR sequence, the two observers assess, for each sequence and lesion, variable semiological aspects assigned as following:

1. Mesial temporal lobe sclerosis:

A. Visual evaluation: the examiners evaluate for hippocampal hyperintensity, compared to the contralateral side.

B. Quantitative assessment: Measuring signal intensities of both hippocampi on the coronal DIR, FLAIR, and T2 images, round small regions of interest
(ROIs) of similar size are manually drown on bilateral hippocampi, and signal intensities are acquired independently at the head, body, and tail levels of the hippocampus. The mean value denotes the signal intensity of the whole hippocampus. The ROIs are placed to include as much of the hippocampal tissues as possible while avoiding the adjacent CSF spaces to diminish the effects of partial volume from the CSF. From the measured signal intensities of bilateral hippocampi on the coronal DIR, FLAIR, and T2 images, the signal-to-noise ratio (SNR), contrast-to-noise ratio (CNR), contrast ratio (CR), and asymmetry index (AI) are calculated for all patients.

$$
\begin{aligned}
& S N R=S I_{1} / S D_{n o} \\
& C N R=\left|S I_{1}-S I_{2}\right| / S D_{n o}, \\
& C R=\left|S I_{1}-S I_{2}\right| / S I_{2} \\
& A I=100(\%) \times\left|S I_{1}-S I_{2}\right| /\left[\left(S I_{1}+S I_{2}\right) / 2\right] \\
& \text { where } S I_{1} \text { is the signal intensity of affected hip- } \\
& \text { pocampus, } \\
& S I_{2} \text { is the signal intensity of contralateral hip- } \\
& \text { pocampus. } \\
& S D_{n o} \text { is the SD of the noise, }
\end{aligned}
$$

2. Focal cortical dysplasia:

A. Visual evaluation: the examiners evaluate for each sequence and lesion, variable semiological aspects assigned as following:

1. Cortical Thickness (CT): examiners evaluate the lesion for cortical thickness comparing it to the contralateral healthy side and giving a result of no difference or increased cortical thickness (CT).

2. Cortical Signal Intensity (CSI): the examiners evaluate a given lesion, compared with contralateral healthy side, giving a result of no difference or CSI change (hypointensity on T1 sequence and hyperintensity on T2, FLAIR and DIR sequences)

3. GM/WM junction blurring: the examiners evaluate whether blurring is present, or not.

4. Subcortical WM hyperintensity: the examiners evaluate if white matter subcortical hyperintensity is detected, or not.

B. Quantitative assessment: calculating average signal intensities of the lesions manually on the coronal DIR, FLAIR, and T2 images. For sake of comparison, the contralateral side for each lesion is further segmented and average intensity is measured. Then the signal-to-noise ratio (SNR), contrast to- noise ratio (CNR), contrast ratio (CR), and asymmetry index (AI) are calculated.

3. Lesions for visual analysis only: 
A. Heterotopia / Polymicrogyria / Schizencephaly: the examiners are asked to evaluate each lesion according to visibility, each lesion is classified as better, similar, or lower visible between DIR versus T1, T2 and FLAIR sequences.

B. Tuberous sclerosis:

1. Counting of cortical tubers: the examiners are asked to count all the lesions on T2, FLAIR and DIR, each sequence will be assessed separately from the others, starting by $\mathrm{T} 2$ sequence followed by the FLAIR and ending by the DIR.

2. Lesion visibility: the examiners are asked to evaluate each lesion according to its visibility, where each lesion is classified as better, similar, or lower visible between DIR versus T2 and FLAIR sequences.

3. GM/WM junction blurring: the examiners are asked to evaluate if blurring is present, or not.

\section{Other lesions:}

The examiners are asked to evaluate each lesion according to its visibility, where each lesion is classified as better, similar, or lower visible between DIR versus T2 and FLAIR sequences.

\section{Statistical analysis}

The statistical analysis of lesions on the different pulse sequences will be performed patient and lesion wise. All data will be gathered, statistically analyzed, and tabulated. Qualitative data will be described using number and percent. Quantitative data will be described using mean and standard deviation. Significance of the obtained results will be judged at the $5 \%$ level ( $P$ value of $<0.05$ was considered statistically significant). Significance between periods was done using Post Hoc Test (adjusted Bonferroni) comparing between different periods using $\mathrm{F}$ test (ANOVA).

\section{Results}

In our study 10 patients (40\%) had mesial temporal sclerosis, 6 patients $(24 \%)$ had focal cortical dysplasia, 1 patient (4\%) had gray matter heterotopia, 1 patient (4\%) had polymicrogyria, 2 patients (8\%) had tuberous sclerosis and 5 patients $(20 \%)$ had gliotic changes. The sociodemographic data are shown in Table 1.

1. Assessment of patients with mesial temporal sclerosis:

In the current study we assessed ten (10) patients with mesial temporal sclerosis with their mean age about 22.9 years (ages ranging between 17 and 35 years).

\section{A. Visual evaluation:}

On T2, only six cases (60\%) showed hyperintense signal in the affected hippocampus, compared to the contralateral side, the cortex and basal ganglia. While on FLAIR and DIR the hyperintense signal is detected within the affected hippocampus in all the ten cases (100\%) compared to the contralateral side, the cortex and basal ganglia (Fig. 1)

B. Quantitative assessment: the measured signal intensity was found to be higher in the ipsilateral side compared to the contralateral side in all the three sequences in $100 \%$ of cases.

Also, all the four contrast parameters (as shown in Table 2) were found to be significantly higher in DIR than in FLAIR and T2 sequences ( $P$ value of $<0.05$ was considered statistically significant).

2. Assessment of patients with focal cortical dysplasia: In the current study we assessed six patients with focal cortical dysplasia with their mean age about 11 years (ages ranging between 1 and 21 years).

A. Visual evaluation:

1. Cortical Thickness: in T1, T2 and FLAIR, only 4 out of the 6 cases (67\%) showed increased cortical thickness compared to all the 6 cases (100\%) on DIR showing increased cortical thickness (Fig. 2)

2. Cortical Signal Intensity: T1 showed cortical signal intensity change in only 2 of the cases (34\%). While T2 and FLAIR, each showed cortical signal intensity change in 4 cases $(67 \%)$ compared to 6 cases $(100 \%)$ on DIR.

3. GM/WM junction blurring: none of the cases (0\%) showed grey-white matter junction blurring in the T2 sequence, compared to 2 out of the 6 cases (34\%) in the remaining other sequences (T1, FLAIR and DIR) whom showed blurring in the grey/white matter junction.

iv. Subcortical WM signal: none of the cases $(0 \%)$ showed subcortical white matter signal change on T1 sequence, compared to 2 cases (34\%) that showed subcortical white matter hyperintense signal on T2, FLAIR and DIR.

B. Quantitative analysis:

The measured signal intensity was found to be higher in the affected side compared to the contralateral side on T2, FLAIR and DIR in 100\% of cases. The contrast parameters are shown in Table 3.

3. Assessment of patients with Heterotopia / Polymicrogyria / Schizencephaly:

We found only one patient in our study having polymicrogyria. The abnormality was lower visualized 

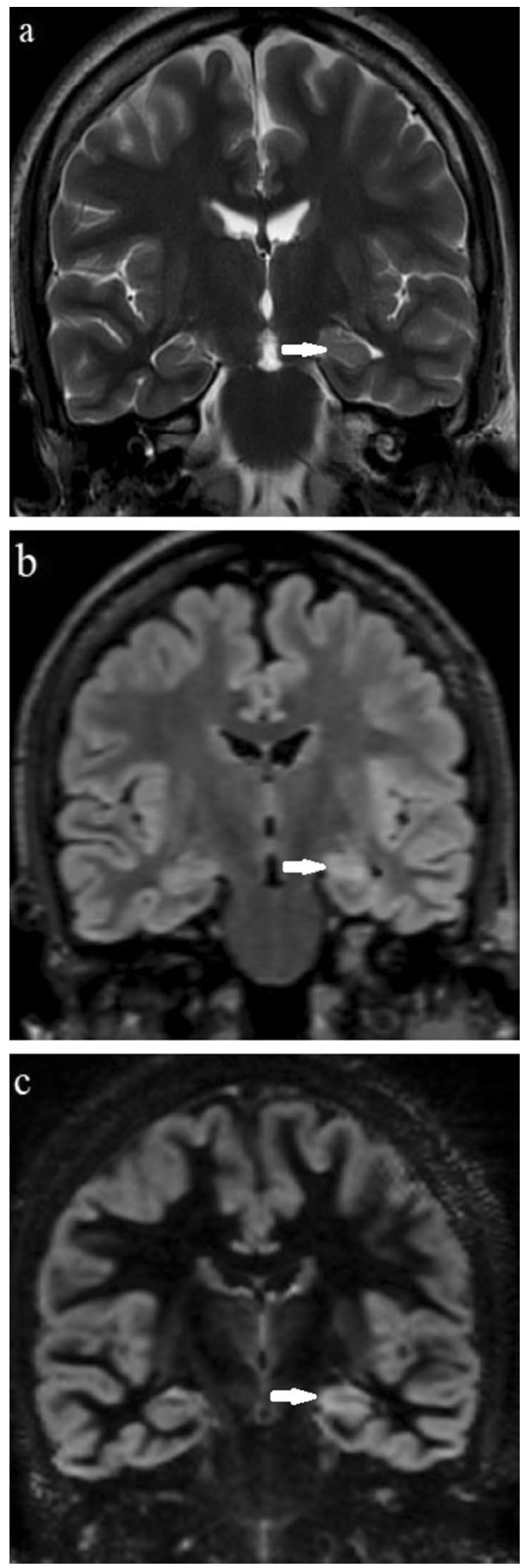

Fig. 1 Left mesial temporal sclerosis in an 18 years old female patient. Oblique coronal T2 weighted (a), FLAIR (b) and DIR (c) images. The left hippocampus is atrophic showing hyperintense signal in T2 (arrow in a), FLAIR (arrow in b) and in DIR (arrow in c). Asymmetry of signal intensity of the hippocampi is most conspicuous on the DIR image
Table 1 Distribution of the studied cases according to demographic data

\begin{tabular}{lll}
\hline & No & $\%$ \\
\hline Sex & 7 & \\
Male & 18 & 72 \\
Female & & \\
Age & $1-57$ & \\
Range & $21.1 \pm 12.5$ & \\
Mean \pm SD & 18 & \\
Median & & \\
\hline
\end{tabular}

Table 2 contrast measurements in patients with MTS compared between DIR, FLAIR, and T2 MRI (Data expressed as means (SDs))

\begin{tabular}{llllll}
\hline Parameter & DIR & FLAIR & T2 & p1 & \multicolumn{1}{c}{$p \mathbf{2}$} \\
\hline SNR & 135.4 & 101.2 & 63.27 & $<0.05^{*}<0.05^{*}$ \\
CR & $(15.01)$ & $(34.21)$ & $(11.45)$ & & \\
CNR & $0.45(0.05)$ & $0.11(0.03)$ & $0.12(0.02)$ & $<0.05^{*}<0.05^{*}$ \\
Al & $41.31(2.32)$ & $9.20(3.34)$ & $6.57(1.92)$ & $<0.05^{*}<0.05^{*}$ \\
\hline
\end{tabular}

$p: p$ value for $F$ test (ANOVA) with repeated measures for comparing between different period

Significance between periods was done using Post Hoc Test (adjusted Bonferroni)

$p 1: p$ value for comparing between DIR and FLAIR

$p 2: p$ value for comparing between DIR and T2

*Statistically significant at $p \leq 0.05$

on DIR than on T1, similar to that on T2 and better than on FLAIR (Fig. 3).

Also, we found a single patient with grey matter heterotopia. The abnormality was almost equally visualized on DIR, T1 and FLAIR, and better visualized on DIR than on T2 (Fig. 4).

4. Assessment of patients with tuberous sclerosis: In our study we found only two patients with tuberous sclerosis.

1. Counting cortical tubers: counting the lesions in the two patients revealed a single lesion seen on DIR which was missed on T2 and FLAIR sequences. Other than that, there were another 18 lesions seen on T2, FLAIR and DIR sequences.

2. Lesions visibility: The lesions were classified as having better, similar, or lower visibility between DIR versus T2 and FLAIR sequences as shown in Table 4.

3. GM/WM junction blurring: grey-white matters junction blurring was noted in 8 lesions out of the whole 19 lesions (about 42\%) in each of the T2 and FLAIR sequences. While this number is almost doubled on DIR to be 15 lesions with grey-white matters 

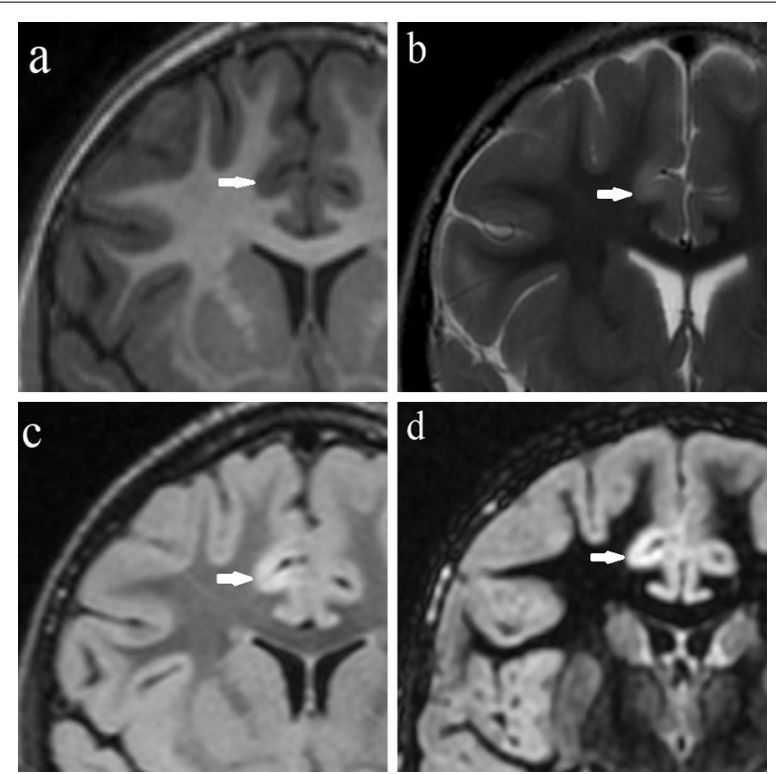

Fig. 2 Focal cortical dysplasia type I in a 9 years old female patient. Coronal T1 (a), T2 (b), FLAIR (c) and DIR (d) images. T1 shows subtle hypointense signal (arrow in a). Also, T2 and FLAIR show increased cortical thickness and hyperintense signal (arrows in $\mathbf{b}, \mathbf{c}$ ). The high signal intensity and increased cortical thickness are most conspicuous on the DIR image (arrow in d)

Table 3 contrast measurements in patients with focal cortical dysplasia compared between DIR, FLAIR, and T2 MRI (Data are presented as means (SDs))

\begin{tabular}{llllll}
\hline Parameter & DIR & FLAIR & T2 & p1 & p2 \\
\hline SNR & $157(45.8)$ & $132(95.7)$ & $127(27.5)$ & $>0.05$ & $>0.05$ \\
CR & $0.32(0.09)$ & $0.09(0.06)$ & $0.11(0.05)$ & $<0.05^{*}$ & $<0.05^{*}$ \\
CNR & $39.5(17.1)$ & $14.5(16.7)$ & $11.9(6.2)$ & $<0.05^{*}$ & $>0.05$ \\
Al & $27.6(6.5)$ & $9(5.4)$ & $10.1(4.6)$ & $<0.05^{*}$ & $<0.05^{*}$ \\
\hline
\end{tabular}

$p: p$ value for $F$ test (ANOVA) with repeated measures for comparing between different period

Significance between periods was done using Post Hoc Test (adjusted Bonferroni)

$p 1: p$ value for comparing between DIR and FLAIR

$p 2: p$ value for comparing between DIR and T2

*Statistically significant at $p \leq 0.05$

junction blurring out of the 19 lesions seen in the two patients (about 79\%) (Fig. 5).

5. Assessment of patients with gliosis.

In our study, there were five patients showing gliosis. The results of their visual assessment were demonstrated in Table 5.
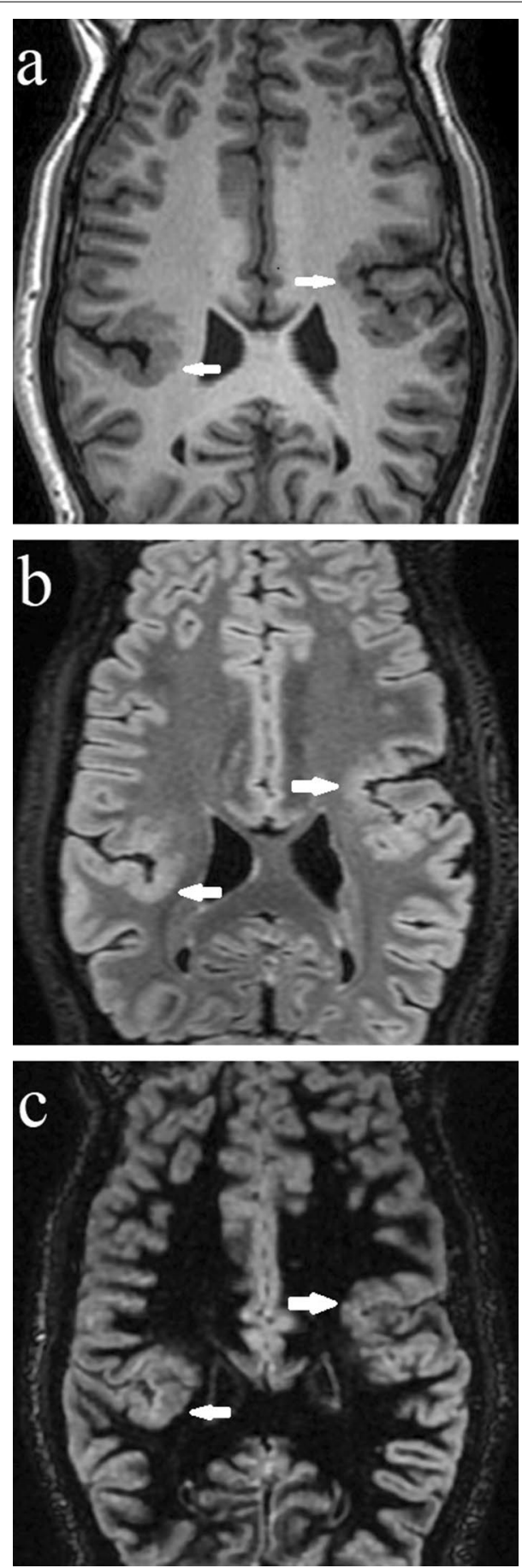

Fig. 3 Bilateral peri-Sylvian polymicrogyria in an 18 years old female patient. Reconstituted curved axial T1 (a), FLAIR (b) and DIR (c) images. Bilateral peri-Sylvian areas of abnormal gyral pattern with irregular convoluted thick cortices noted (arrows in $\mathbf{a}-\mathbf{c}$ ). No signal intensity difference between the normal and abnormal cortex on any of the pulse sequences, including DIR 


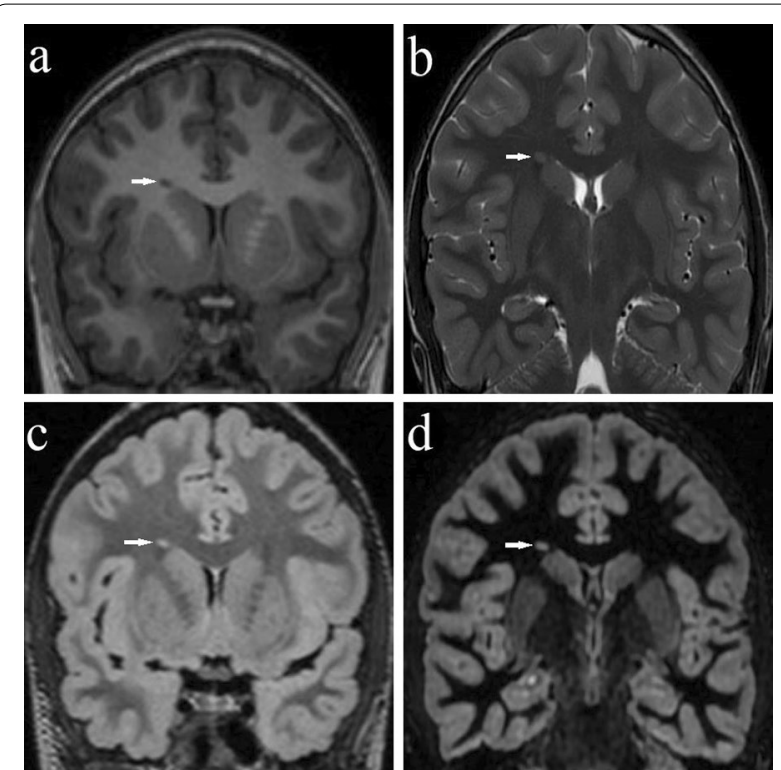

Fig. 4 Right centrum semi-ovale nodular heterotopia in a 12 years old female patient. Coronal MPGRET1 (a), T2 (b), FLAIR (c) and DIR (d) images. A heterotopic grey matter nodule noted following the signal of the grey matter in all pulse sequences (arrows in $\mathbf{a}-\mathbf{d}$ ), however, most conspicuous on 3DT1 and DIR

\section{Discussion}

In the visual assessment of mesial temporal sclerosis (MTS), there was a characteristic high signal intensity of the affected hippocampus in patients with MTS on DIR images, which was confirmed by the quantitative evaluation (in the form of SNR, CR, CNR and AI shown in Table 2. Our study agreed with what Qiong et al. [6] stated in their study, that DIR images can describe hippocampal sclerosis (HS) with high signal noise to ratio (SNR) and contrast to noise ratio (CNR), superior to conventional MR sequences (T2 and FLAIR sequences). However, our study showed significant increase in the contrast ratio (CR) and asymmetry index (AI) as well.

In case of focal cortical dysplasia, our study showed that the 3D DIR was capable to increase the detectability of some of the focal cortical dysplasia features, increased cortical thickness and cortical signal intensity in particular. Ryan [10] stated that the sensitivity and specificity of the DIR sequence for FCD among their observers ranged from 50 to $88 \%$ and $67-91 \%$ respectively and reached to conclusion that the DIR sequence is sensitive for the detection of focal cortical dysplasia, particularly when reviewed by experienced interpreters. Other researchers as Granata et al. [11] concluded that the main advantage of DIR acquisition could be its capability to demonstrate at the same time all the semiological characteristics of cortical development disorders.
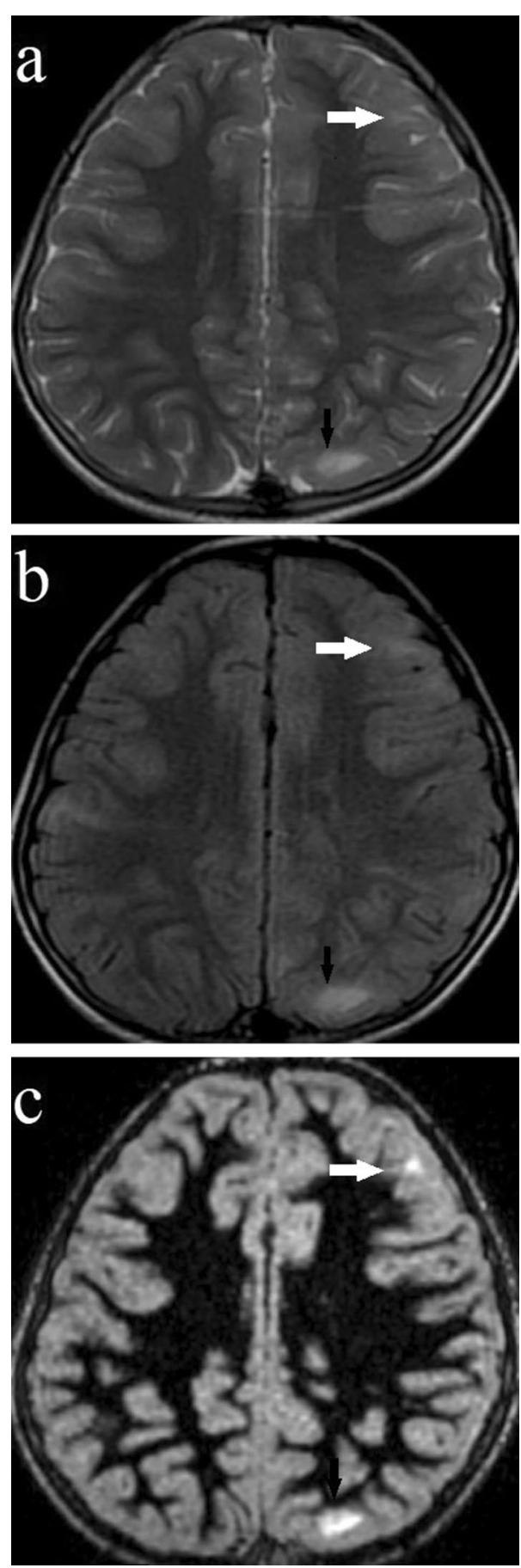

Fig. 5 Tuberous Sclerosis in an 8 years old female patient. Axial T2 (a), FLAIR (b) and DIR (c). A sub-cortical tuber in the left frontal lobe shows very subtle hyperintense signal in $\mathrm{T} 2$ and FLAIR (white arrows in $\mathbf{a}, \mathbf{b}$ ) while extreme high signal is noted on DIR (white arrow in $\mathbf{c}$ ). Another sub-cortical tuber is seen left posterior parietal lobe (black arrows in $\mathbf{a}-\mathbf{c}$ ) 
Table 4 Classify the lesions of tuberous sclerosis according to visibility in DIR compared to T2 and FLAIR

\begin{tabular}{llll}
\hline Sequence & Better & Similar & Lower \\
\hline T2 & $10(53 \%)$ & $9(47 \%)$ & $0(0 \%)$ \\
FLAIR & $14(73 \%)$ & $3(16 \%)$ & $2(11 \%)$ \\
\hline
\end{tabular}

Table 5 classifying visually assessed lesions on patients with gliosis according to visibility on DIR compared to the T2 and FLAIR

\begin{tabular}{llll}
\hline Sequence & Better & Similar & Lower \\
\hline T2 & $4(80 \%)$ & $1(20 \%)$ & $0(0 \%)$ \\
FLAIR & $0(0 \%)$ & $4(80 \%)$ & $1(20 \%)$ \\
\hline
\end{tabular}

In assessment of tuberous sclerosis patients, one of the cortical tubers was missed on T2 and FLAIR and only initially visualized on DIR. Also most of the lesions were better visualized on DIR than on FLAIR sequence. This can reflect the higher capability of the DIR sequence in lesion detectability over the T2 and FLAIR sequences. In the study by Cotton et al.[12] they found that in all their patients, the cortical tubers appeared very bright on the DIR sequence and were well outlined compared with high resolution T2 or FLAIR images and they concluded that DIR images may have a complementary role in the MRI evaluation of the tuberous sclerosis patients.

\section{Conclusion}

Our study concluded that the greatest value of the DIR sequence is its higher ability in detecting multiple characteristics of the lesions in a one sequence. Also, it should be clear that every MRI sequence has its value and the DIR is not to replace any of them, but to add to them in the sake of patient's benefit.

\section{Abbreviations}

Al: Asymmetry index; CNR: Contrast-to-noise ratio; CR: Contrast ratio; CSI: Cortical signal intensity; CT: Cortical thickness; DIR: Double inversion recovery; FCD: Focal cortical dysplasia; FOV: Field of view; HS: Hippocampal sclerosis; MCD: Malformations of cortical development; MTS: Mesial temporal sclerosis; TE: Echo time; TI: Inversion time; TR: Repetition time; TSc: Tuberous sclerosis; SNR: Signal-to-noise ratio.

\section{Acknowledgements}

Not applicable.

\section{Authors' contributions}

MA contributed in the design of the work; the acquisition, analysis and interpretation of data and wrote the manuscript. TS contributed in the design of the work; the analysis and interpretation of data and supervised the work. AE contributed in the design of the work; and interpretation of data and supervised the work. MM contributed in the design of the work; the acquisition, analysis, and interpretation of data and supervised the work. All authors approved the final manuscript.
Funding

No funding was received for this work.

\section{Availability of data and materials}

All the data that this work depended on to reach the conclusion are available and to be delivered when requested.

\section{Declarations}

Ethics approval and consent to participate

The study was approved by the Unified Standards Committee of University of Alexandria. (committee's reference number is not available). As a retrospective study, confidentiality of records is considered.

\section{Consent for publication}

Not applicable.

Competing interests

The authors declare that they have no competing interests.

\section{Author details}

${ }^{1}$ Department of Radiodiagnosis, Faculty of Medicine, University of Alexandria, Alexandria, Egypt. ${ }^{2}$ Department of Neuropsychiatry, Faculty of Medicine, University of Alexandria, Alexandria, Egypt.

Received: 23 March 2021 Accepted: 11 September 2021

Published online: 29 September 2021

\section{References}

1. Falco-Walter JJ, Scheffer IE, Fisher RS (2018) The new definition and classification of seizures and epilepsy. Epilepsy Res 1(139):73-79

2. Von Oertzen J, Urbach $H$, Jungbluth $S$, Kurthen $M$, Reuber M, Fernandez G, Elger CE (2002) Standard magnetic resonance imaging is inadequate for patients with refractory focal epilepsy. J Neurol Neurosurg Psychiatry 73(6):643-647

3. Gho SM, Nam Y, Zho SY, Kim EY, Kim DH (2010) Three dimension double inversion recovery gray matter imaging using compressed sensing. Magn Reson Imaging 28:1395-1402

4. Zhang Q, Li Q, Zhang J, Zhang Y (2011) Double inversion recovery magnetic resonance imaging (MRI) in the preoperative evaluation of hippocampal sclerosis: correlation with volumetric measurement and proton magnetic resonance spectroscopy (1H MRS). J Comput Assist Tomogr 35(3):406-410

5. Barkovich AJ, Dobyns WB, Guerrini R (2015) Malformations of cortical development and epilepsy. Cold Spring Harb Perspect Med 5(5):a022392

6. Li Q, Zhang Q, Sun H, Zhang Y, Bai R (2011) Double inversion recovery magnetic resonance imaging at $3 \mathrm{~T}$ : diagnostic value in hippocampal sclerosis. J Comput Assist Tomogr 35(2):290-293

7. Desikan RS, Barkovich AJ (2016) Malformations of cortical development. Ann Neurol 80(6):797-810

8. Parazzini C, Triulzi F (2016) Malformations of cortical development. In: Perinatal neuroradiology. Springer, Milano

9. Soares BP, Porter SG, Saindane AM, Dehkharghani S, Desai NK (2016) Utility ofdouble inversion recovery MRI in paediatric epilepsy. Br J Radio 89(1057):20150325

10. Ryan ME (2016) Utility of double inversion recovery sequences in MRI Pediatr Neurol Briefs 30(4):26

11. Granata F, Morabito R, Mormina E, Alafaci C, Marino S, Laganà A, Vinci SL, Briguglio M, Calamuneri A, Gaeta M, Salpietro V (2016) 3T double inversion recovery magnetic resonance imaging: diagnostic advantages in the evaluation of cortical development anomalies. Eur J Radiol 85(5):906-914

12. Cotton F, Rambaud L, Hermier M (2006) Dual inversion recovery MRI helps identifying cortical tubers in tuberous sclerosis. Epilepsia 47(6):1072-1073

\section{Publisher's Note}

Springer Nature remains neutral with regard to jurisdictional claims in published maps and institutional affiliations. 